\title{
TV/Series
}

$2 \mid 2012$

Les séries télévisées dans le monde : Échanges,

déplacements et transpositions

\section{Les Invincibles en France : Temps et espace d'une adaptation}

Justine Huet et Sathya Rao

\section{OpenEdition}

\section{Journals}

Édition électronique

URL : http://journals.openedition.org/tvseries/1452

DOI : 10.4000/tvseries. 1452

ISSN : 2266-0909

Éditeur

GRIC - Groupe de recherche Identités et Cultures

Référence électronique

Justine Huet et Sathya Rao, "Les Invincibles en France : Temps et espace d'une adaptation »,TV/ Series [En ligne], 2 | 2012, mis en ligne le 01 novembre 2012, consulté le 05 mai 2019. URL : http:// journals.openedition.org/tvseries/1452; DOI : 10.4000/tvseries.1452

\section{(c) (i) $\odot$}

TV/Series est mis à disposition selon les termes de la licence Creative Commons Attribution - Pas d'Utilisation Commerciale - Pas de Modification 4.0 International. 


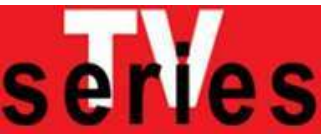

Les Invincibles en France : Temps et espace d'une adaptation'

Justine HUET

Sathya RAO

Après avoir situé la série Les Invincibles dans le contexte du renouveau des séries québécoises et des échanges télévisuels entre la France et le Québec, cet article s'attache à la comparer à l'adaptation française qui en a été faite en 2010 par Arte et la société de production Making Prod. Deux axes génériques de comparaison seront privilégiés, à savoir la représentation du temps et de l'espace. Ces axes nous permettront de rendre compte de plusieurs différences de style entre l'original et l'adaptation par-delà le simple constat des écarts culturels entre la France et le Québec.

$\mathrm{L}$

es séries télévisées sont une véritable institution au Québec. Depuis La Famille Plouffe (Radio-Canada, 1953-1959), Roger de la Garde estime à 850 le nombre de séries télévisées diffusées dans la Belle Province sur une période d'environ $50 \mathrm{ans}^{2}$. D'entre toutes, c'est sans nul doute le téléroman ${ }^{3}$ qui a connu le plus grand succès populaire et suscité le nombre d'études 4 le plus important. Certains téléromans ont ainsi battu des records mondiaux, que ce soit en termes de longévité, de cotes d'écoute ou de parts de marché. Plusieurs raisons expliquent l'attachement particulier des Québécois aux téléromans : la continuité avec une certaine tradition littéraire (notamment la littérature de terroir5), l'ancrage identitaire dans le milieu francophone, la familiarité des téléspectateurs avec les protagonistes des téléromans, souvent issus de milieux populaires,

${ }^{1}$ Les Invincibles, série québécoise diffusée du 14 septembre 2005 au 25 mars 2009 sur RadioCanada, a donné lieu à une adaptation française du même titre, diffusée sur Arte depuis le 9 mars 2010.

${ }^{2}$ Roger de la Garde, "Le téléroman québécois : une aventure américaine », Ciberlegenda, $\mathrm{n}^{\circ} 10$, 2002, http://www.uff.br/mestcii/roger1.htm, consulté le 30 juin 2011.

3 Nous prenons le terme dans son sens général de séries télévisées québécoises de langue française. Cela dit, nous sommes conscients de la nécessité exprimée par Danielle Aubry («Réflexion sur les genres télévisuels», Fréquence/Frequency, n¹1-12, 2004, p. 92-112) et Frédéric Demers ( Téléroman, télésérie, feuilleton... : retour sur une source de confusion sémantique », Communication, vol. $25, \mathrm{n}^{\circ} 1$, 2006, lien internet consulté le 30 juin 2011,

http://communication.revues.org/index1480.html) d'établir une classification plus précise, ne serait-ce que pour rendre compte de l'évolution interne du genre.

${ }^{4}$ Nathalie Nicole Bouchard, "À la recherche des téléromans : revue de la littérature consacrée aux radioromans et aux téléromans québécois ", Communication, vol. 20, $\mathrm{n}^{\circ} 1,2000$, p. 217-248.

5 Plusieurs téléromans, à commencer par La Famille Plouffe, sont des adaptations de romans populaires. 
et enfin le rôle de catalyseur des téléromans en matière de changements sociaux ${ }^{6}$. Comme le souligne Catherine Saouter :

En quarante ans, le téléroman est devenu un des objets culturels significatifs du Québec. Il alimente des carrières d'auteurs et de comédiens, polarise celles de cinéastes et nourrit des aspirations de la jeunesse : on entend, dans les classes universitaires, les étudiants d'études littéraires révéler que leur vœu le plus cher est d'apprendre à scénariser des téléromans7.

Se situant eux-mêmes au croisement de plusieurs genres (romans, radioromans, sitcoms étasuniens), les téléromans sont donc à la fois dépositaires d'une certaine tradition nationale ${ }^{8}$ et permettent d'aborder des questions d'actualité parfois sensibles9.

\section{Le renouveau des séries télévisées québécoises}

Depuis la fin des années 1980, on assiste à un renouveau des séries télévisées québécoises qu'illustre le succès international de Lance et compte (Radio-Canada, 1986-1989), Un gars, une fille (Radio-Canada, 1997-2003), Rumeurs (Radio-Canada, 2002-2008), Les Bougon (RadioCanada, 2004-2006), Les Hauts et les bas de Sophie Paquin (RadioCanada, 2006-2009) ou, tout dernièrement, Les Parent (Radio-Canada 2008-). On sort désormais de l'intimité des studios pour filmer en extérieur. Le ton se fait plus critique, le propos volontiers grivois voire parfois politiquement incorrect. La narration perd en linéarité pour se complexifier et explorer des formats plus courts. Quant à l'esthétique, elle rompt avec le réalisme des téléromans comme Virginie (Radio-Canada, 1996-2010), L'Auberge du chien noir (Radio-Canada, 2003-), Annie et ses hommes (TVA, 2002-2009) pour emprunter volontiers à différents genres. En outre, la responsabilité de l'œuvre n'est plus uniquement le fait de télédiffuseurs publics comme Radio-Canada, elle concerne désormais des producteurs indépendants qui doivent rivaliser d'originalité et de moyens pour promouvoir leurs réalisations. Les Invincibles illustre bien ce

${ }^{6}$ Véronique Nguyên-Duy, « Le téléroman et la volonté d'une télévision originale », in Variations sur l'influence culturelle américaine, éd. Florian Sauvageau, Sainte-Foy, Québec, Les Presses de l'Université Laval, 1999, p. 131-158.

7 Catherine Saouter, «Le téléroman, art de nouveaux conteurs : formes et influences du récit téléromanesque », Recherches sociographiques, vol. 33, n² 2, 1992, p. 262 [p. 259-276].

8 Dominic Leroy, "Téléroman québécois et identité culturelle nationale ", Études canadiennes, $\mathrm{n}^{\circ} 34,1993$, p. 7-27.

9 Nathalie Nicole Bouchard, «Scoop et les communautés interprétatives : Sémiotique de la réception du téléroman québécois ", Thèse de doctorat en communication, Montréal, UQAM, 1997. 
renouveau : la série fait cohabiter plusieurs niveaux de narration et intègre des éléments tant narratifs qu'esthétiques empruntés à différents genres (cinéma, bande dessinée, science-fiction, mockumentaire) tout en proposant un regard sans concession sur des sujets de société comme l'identité masculine. Ainsi la série met-elle en scène quatre trentenaires, Pierre-Alexandre (François-Xavier dans la version française), Carlos (Hassan dans la version française), Steve (Vince dans la version française) et Rémi (Mano dans la version française), qui décident de nouer un pacte par lequel ils s'engagent à rompre avec leur petite amie du moment pour profiter pleinement de leur célibat. Pour autant, Les Invincibles conserve certains traits typiques des téléromans : importance des relations familiales et amicales, inscription de la diégèse dans le quotidien et mise en scène de personnages ordinaires. Traditionnellement au cœur des téléromans ${ }^{10}$, le thème de la réussite amoureuse se trouve ici détourné puisqu'il s'agit de fonder un pacte d'amitié masculin précisément sur le rejet de tout engagement amoureux. Les Invincibles apparaît donc comme un produit hybride où les influences thématiques du téléroman québécois et de sitcoms étasuniennes comme Friends (NBC, 1994 -2004) et Seinfeld (NBC, 1989-1998) se combinent à une esthétique sophistiquée qui emprunte visuellement à la fois au cinéma hollywoodien, aux comics Marvel $^{11}$ et à des séries comme 24 (24 Heures chrono, Fox, 2001-2010) et The Office (NBC, 2005-(12. Comme l'observe Frédéric Demers, l'hybridité propre à un grand nombre de séries créées ces dix dernières années au Québec (et ailleurs), se traduit par une difficulté à proposer une typologie appropriée quand il s'agit de les caractériser :

Il convient en terminant de noter que la fiction sérielle québécoise a commencé, au tournant du siècle, à amalgamer de plus en plus les trois genres que nous venons de définir. Les scènes extérieures se sont multipliées dans les fictions sérielles qui appartiennent au genre téléromanesque, bien qu'elles y demeurent encore assez peu nombreuses. Il n'y a pas si longtemps, seules les téléséries et les miniséries étaient filmées en cinéma, mais des œuvres pourtant plus près du téléroman que de la télésérie par la narration (Caserne 24, Rumeurs) sont venues modifier cette pratique. Parodiant l'univers mélodramatique des soap-operas étatsuniens, l'émission Le cœur a ses raisons serait à situer à la jonction du téléroman, par son rythme et ses intrigues simultanées, et, par l'invraisemblance des protagonistes et de leurs

10 Nathalie Goudreau, «Les téléromans au Québec en 1993-1994 et 1994-1995. Contenu, conditions de production et auditoire ", Communication, vol. 18, $\mathrm{n}^{\circ} 1,1997, \mathrm{p} .125-136$.

${ }_{11}$ À l'instar de la série Heroes (NBC, 2006-2010), Les Invincibles déconstruisent la figure mythique du super héros.

12 Nous faisons référence ici à la version étasunienne mieux connue du public québécois que la série originale britannique. 
péripéties, de la caricaturale comédie de situation. Quant à Minuit le soir, l'humour noir et la facture visuelle trashy qui lui sont caractéristiques en font, là encore, une œuvre à part et, pour cette raison, difficile à classer nettement ${ }^{13}$.

Cette hybridité affecte Les Invincibles qui, si l'on se base sur les catégories proposées par Frédéric Demers dans le même article, appartiendrait à la fois au genre de la «télésérie » en raison de sa facture cinématographique et à celui de la « comédie de situation » du fait de sa trame narrative.

Jean-François Rivard, le réalisateur des Invincibles, et Podz, à qui l'on doit notamment Minuit, le soir (Radio-Canada, 2005-2007; diffusée sur France 2 en 2009), sont parmi les principaux artisans de ce renouveau des séries télévisées québécoises ${ }^{14}$. Connaissant bien l'un comme l'autre le monde du cinéma ${ }^{15}$, les deux réalisateurs revendiquent explicitement l'influence de leur culture cinématographique sur leur œuvre télévisuelle. Aux yeux de Podz, « [...] il n'y a pratiquement pas de différence ; j'approche la réalisation télé en cinéaste, et je ne vois pas comment je pourrais faire autrement ${ }^{16}$ ». Cette conception n'est pas sans rappeler l'intérêt de cinéastes de talent comme Steven Spielberg et Sidney Lumet pour les séries télévisées. On pense également à David Lynch, créateur, avec Mark Frost, de la série Twin Peaks (ABC, 1990-1991), à Martin Scorsese, qui a réalisé le premier épisode de Boardwalk Empire (HBO, 2010-), ou encore à Frank Darabont, qui a adapté la bande dessinée The Walking Dead (AMC, 2010-) pour le petit écran. Dans le contexte québécois, Catherine Saouter reconnait également la porosité de la frontière entre télévision et cinéma, en particulier pour ce qui concerne la production et la réalisation de téléromans comme Lance et compte et Les Filles de Caleb (Radio-Canada, 1990) ${ }^{17}$, qui bénéficient des moyens et de l'expertise de producteurs indépendants ${ }^{18}$. Comme nous le verrons, il en est de même pour Les

13 Demers, $\S 18$.

14 En outre, Podz (de son vrai nom Daniel Grou) s'est récemment vu confier la réalisation de la série française Xanadu, dont le premier épisode a été diffusé sur Arte le 30 avril 2011. Il a également réalisé des épisodes de plusieurs séries canadiennes anglophones, comme Big Wolfon Campus (1999-2002) et Vampire High (2001-2002), diffusées par le réseau YTV, et de The Hunger (Showtime, 1997-2000), coproduite par la Grande-Bretagne et le Canada.

15 Jean-Francois Rivard est l'auteur de plusieurs courts-métrages. Quant à Podz, il a réalisé à ce jour deux longs métrages : 101/2 (2010) et Les Sept Jours du talion (2010).

${ }^{16}$ Pierre Barrette, "L'état de la série télé au Québec : petite enquête en forme de dialogues », 24 images, $\mathrm{n}^{\circ} 138,2008$, p. 20 [p. 18-20].

${ }^{17}$ Saouter, p. 263.

${ }^{18}$ Selon Nathalie Goudreau, les budgets des producteurs autonomes sont en général beaucoup plus importants que ceux des télédiffuseurs, ce qui permet des conditions de tournage proches de celles de films (p. 128). 
Invincibles qui, en plus de profiter d'un budget et de moyens relativement importants, multiplie les références au cinéma américain des années 198090 et recourt fréquemment à des techniques cinématographiques.

Non seulement les nouvelles séries québécoises puisent abondamment dans l'univers cinématographique, mais elles accordent une place centrale à la réalisation. À cet égard, comme Jean-François Rivard l'admet volontiers :

[La télévision québécoise] a longtemps été le fait des auteurs ; le scénariste était la véritable « vedette » et le réalisateur s'effaçait plus ou moins derrière le texte. Ce sont des gens comme Podz, Louis Choquette (Temps dur, Jack Carter) et Patrice Sauvé (La vie, la vie, Grande Ourse) qui ont changé les choses. C'est eux qui m'ont donné le goût de faire de la télévision ${ }^{19}$.

Cette perspective déplace l'accent de la scénarisation traditionnellement au centre des téléromans (en particulier lorsque celle-ci était confiée à des écrivains de renom comme Victor-Lévy Beaulieu, Marcel Dubé, Guy Dufresne, Roger Lemelin, Pierre Gauvreau et Robert Choquette) vers la dimension proprement cinématographique et technique de la réalisation. Dans le cas de la série Les Invincibles, le réalisateur Jean-François Rivard partage la tâche de scénarisation avec François Létourneau, plus connu comme dramaturge, qui campe également le rôle de P-A dans la série ${ }^{20}$. Cette pratique scénaristique en binôme, fréquente dans le téléroman ${ }^{21}$, contraste avec le modèle étasunien reposant, lui, sur le travail en équipe de scénaristes professionnels. En contrepartie, l'imbrication étroite des niveaux de la scénarisation, de la réalisation et de la mise en scène aurait tendance à faciliter la direction d'acteur, de même que l'intégration des contraintes filmiques dans l'écriture du scénario. La version française a également pris le parti de contrevenir à la division traditionnelle des tâches en confiant à Brigitte Bémol et à Bertrand Marzec, tous deux coscénaristes, les rôles respectifs de Meike et de Martin. D’une manière générale, il semble que le renouveau des séries télévisées québécoises passe, en premier lieu, par une recherche visuelle plus audacieuse, nourrie en partie par une proximité plus grande avec le cinéma. Jean-François Rivard va jusqu'à parler de l'émergence d'une «télévision d'auteur ${ }^{22}$ » capable non

19 Barrette, p. 20.

20 À cet égard, on peut également noter que la chanteuse Kim Bingham, qui a composé et interprété le générique de la série, tient le rôle de la chanteuse du groupe Skydome dans la série. ${ }^{21}$ Nguyên-Duy, p. 137.

${ }^{22}$ Barrette, p. 20. On ne peut s'empêcher d'établir un parallèle avec le cinéma d'auteur porté par la Nouvelle Vague. Le terme "télévision d'auteur» a cependant été utilisé dans un contexte différent pour parler de l’incursion de cinéastes européens de renom comme Rossellini, Ruiz et 
seulement de concurrencer les productions française et étasunienne sur le marché intérieur, mais aussi de s'exporter hors des frontières du Québec malgré les nouvelles contraintes financières qui pèsent sur elles ${ }^{23}$. En France, on assiste à une évolution similaire, en particulier dans le genre des séries policières, avec des feuilletons haut de gamme comme Pigalle, la nuit (Canal+, 2009) et Braquo (Canal+, 2009-), dont le mode de production, la distribution d'acteurs et la réalisation n'ont rien à envier au cinéma.

\section{Les transferts télévisuels entre la France et le Québec : problématiser l'espace télévisuel franco-québécois}

Depuis Un gars, une fille, créée par Guy A. Lepage et diffusée sur Radio-Canada avant d'acquérir une notoriété internationale, les séries québécoises sont de plus en plus présentes dans le paysage audiovisuel français. Il est intéressant de noter que celles-ci font l'objet de modes d'appropriation différents. Tandis que certaines sont adaptées (comme Les Invincibles, ou encore Un gars, une fille et Les Bougon), d'autres sont doublées, à l'instar de Lance et compte et de Minuit, le soir24. Enfin, quelques rares séries sont diffusées en version originale, à l'image de la série parodique Le cour a ses raisons (TVA, 2005-2007), présente sur la grille 2008 des programmes de NRJ 12. Le maintien de la version québécoise constitue à l'évidence un risque que les chaînes ne sont pas prêtes à prendre, même dans leur programmation de fin de soirée, à moins que la langue et les références culturelles n'aient été sciemment aseptisées dans la série originale elle-même, comme c'est le cas dans Le coeur a ses raisons, pour les besoins de la parodie. Le français exagérément soutenu parlé par Criquette Rockwell, Brad Montgomery et les autres protagonistes de la série se rapproche (y compris au niveau de la prononciation) de la forme normative dans laquelle les soaps étasuniens sont doublés au Québec. Dans ce cas particulier, l'effet de parodie, qui échappe très probablement au public français, permet de faire l'économie du doublage

Rohmer dans le genre documentaire (voir Françoise Berdot, La télévision d'auteur(s), Aléas éditeur, Lyon, 2003-2004).

${ }^{23} \mathrm{La}$ crise « des séries lourdes » au début des années 2000 a forcé les télédiffuseurs tant privés que publics à privilégier des formats de série avec des coûts de production moins importants ou tout simplement à faire l'acquisition de droits de diffusion de séries étasuniennes. En outre, contrairement à des pays comme les États-Unis et même la France, le Québec dispose d'un marché intérieur restreint, ce qui limite considérablement les perspectives de rentabilité financière et, par voie de conséquence, n'encourage pas ou peu les investissements.

${ }^{24}$ La question du doublage en français standard des séries québécoises soulève régulièrement des polémiques dont se font l'écho les blogs de spectateurs. 
du québécois vers le français. En définitive, le format de l'adaptation est étroitement lié aux contraintes économiques et culturelles imposées par le système d'accueil (budget, heure de diffusion, public cible, etc.). Il importe également de préciser que l'échange de programmes télévisuels entre la France et le Québec est loin d'être unilatéral. À l'exportation de séries télévisées québécoises répond notamment celle de concepts d'émissions françaises grand public comme Fort Boyard, La Fureur, Star Academy, Tout le monde en parle et, plus récemment, Les Enfants de la télé. Sur le plan institutionnel existent de nombreuses ententes internationales qui favorisent les coproductions entre la France et le Québec, tant dans le domaine du cinéma que de l'animation et de la télévision²5.

L’importance accrue des transferts de programmes de télévision (et des séries en particulier) entre la France et le Québec justifie la nécessité de problématiser l'espace télévisuel franco-québécois. Dans un article récent portant sur la pratique de la coproduction officielle entre la France et le Québec, Philippe Gauthier pose bien les termes de ce problème :

[...] [C]omment, à l'intérieur d'une coproduction France-Québec, peut-on rendre compte de la complexité de deux cultures simultanément ? [...] Quels sont les mécanismes mis de l'avant pour intéresser et rejoindre deux publics nationaux dont les horizons d'attente ne se chevauchent pas nécessairement ${ }^{26}$ ?

Dans le cas de l'adaptation de séries télévisées québécoises, ces questions se posent de façon sensiblement différente. Outre le fait que le cadre légal et institutionnel n'est pas le même que celui d'une coproduction, l'adaptation consiste moins à faire coexister deux cultures qu'à les substituer l'une à l'autre. Comme l'a montré Linda Hutcheon, l'adaptation est une opération sémiotique complexe qui s'effectue sur une variété de niveaux différents (format, public, contexte, genre, etc.) ${ }^{27}$. Dans le domaine des Television Studies, qui nous concerne plus directement, cette notion connaît des développements particulièrement intéressants sous les traits du concept de "TV program format », élaboré notamment

25 On mentionnera notamment les traités bilatéraux de 1983 et les mini-traités de 1992 qui facilitent l'obtention de financements étatiques. On peut noter également qu'en 2007, la France était le plus important partenaire du Canada en ce qui concerne les coproductions officielles (Commissariat aux langues officielles, Ombres sur le paysage télévisuel canadien : place du français sur les ondes et production en contexte minoritaire, 2009, p. 46, http://www.ocolclo.gc.ca/docs/f/televisionlandscape paysagetelevisuel f.pdf, consulté le 30 juin 2011). Il est intéressant de signaler que ces coproductions se font essentiellement en France.

26 Philippe Gauthier, «Les coproductions cinématographiques France-Québec : un espace culturel problématique. L'exemple de J'ai mon voyage! (Denis Héroux, 1973). Nouvelles 'vues' sur le cinéma québécois », Cinéma québécois, n¹0, 2008-2009, p. 2 [p. 1-16].

${ }^{27}$ Linda Hutcheon, Theory of Adaptation, New York et Londres, Routledge, 2006. 
par Albert Moran ${ }^{28}$. L'intérêt de ce concept est de rendre compte de la circulation globale de produits télévisuels standardisés de tout type (jeux, émissions de divertissement, séries, etc.) entre publics locaux ${ }^{29}$. Tout comme l'adaptation, le «TV program format » est un phénomène complexe qui englobe une variété d'aspects différents allant des contraintes légales à l'acclimatation culturelle du produit. Dans le cas particulier des Invincibles, le concept de "TV program format 》 doit être appliqué avec un certain nombre de mises au point. En premier lieu, la série n'a pas originellement été conçue pour l'exportation comme c'est le cas de certains jeux télévisés, ce qui augmente la marge de créativité des adaptateurs mais renforce la difficulté de l'adaptation. En second lieu, elle concerne des espaces partageant un certain nombre de référents culturels et linguistiques. À cet égard, l'adaptation des Invincibles peut être rapprochée de celle dont la série britannique The Office (BBC, 2001-2003) a fait l'objet aux États-Unis ${ }^{30}$. Plutôt que d'exposer le cadre général des transferts télévisuels entre la France et le Québec, nous privilégierons l'étude spécifique des éléments stylistiques (tournage, montage, scénographie), culturels, et intertextuels (nature du programme, codes télévisuels) de l'adaptation ${ }^{31}$, tout en étant bien conscients que ceux-ci sont parfois directement liés à des considérations juridiques et économiques. Ce faisant, notre propos sera de montrer que, dans le cas de la série Les Invincibles, le travail d'adaptation consiste tout autant à refléter la réalité culturelle et nationale du public cible ${ }^{2}$ qu'à opérer des choix stylistiques particuliers. Ceux-ci contribuent à faire de l'adaptation un produit singulier, avec ses qualités et ses défauts, plutôt qu'une simple copie.

\section{La question du temps}

Nous tenons à préciser d'emblée que notre étude comparative ne portera que sur la première saison de la série originale et de son adaptation

${ }^{28}$ Albert Moran, TV Formats Worldwide: Localizing Global Programs, Bristol et Wilmington, Intellect Books, 2009.

29 Moran, p. 115-116.

$3^{\circ}$ Voir Alexandra Beeden et Joost de Bruin, "The Office: Articulations of National Identity in Television Format Adaptation ", Television \& New Media, vol. 11, nº1, juin 2010, p. 3-19. Voir également l'article de Shannon Wells-Lassagne dans le présent numéro de TV/Series.

${ }^{31} \mathrm{Ce}$ modèle d'analyse tripartite proposé par Albert Moran a le mérite de couvrir l'adaptation dans la diversité de ses dimensions.

${ }^{2}$ C'est en général cette approche «culturaliste» qui est privilégiée par bon nombre de chercheurs. L'inconvénient d'une telle approche est qu'elle s'appuie parfois sur une représentation stéréotypée ou idéalisée des cultures source et cible. Faute d'une solide connaissance de ces cultures, le chercheur risque de conforter des clichés culturels et sociaux. 
française 33 . Certes très générale, la question du temps sera envisagée, dans le cadre de cette étude, de quatre façons différentes mais complémentaires.

\section{a) Cibler ou ne pas cibler les trentenaires}

Dans plusieurs entretiens, les scénaristes québécois des Invincibles, François Létourneau et Jean-François Rivard, se défendent d'avoir voulu faire le portrait de leur génération ${ }^{34}$, même si c'est de cette façon que beaucoup ont compris le propos de la série35. Ils disent plutôt avoir cherché à écrire de «bonnes histoires " susceptibles de plaire au public sans distinction d'âge. Ce n'est pas l'interprétation des producteurs français, Stéphane Drouet et Matthieu Viala, qui ont choisi, eux, de mettre l'accent sur les problèmes des trentenaires, " de cette génération d'adultes qui se cherchent, un peu immature ${ }^{36} »$. Incarnée par le personnage de Tanguy, héros du film éponyme d'Étienne Chatiliez (2001), cette génération a reçu une cohorte d'appellations : " bof », "X », " gloubi-boulga », " précaire », "désenchantée ». Elle a également fait l'objet d'études sociologiques, littéraires, psychanalytiques et, bien sûr, d'études de marché. Les quelques articles de presse français consacrés à la série d'Arte renforcent cette interprétation 37 . On retrouve même le terme d' « adulescent » sous la plume d'un chroniqueur québécois, qui l'utilise à propos de la version française en prenant soin de le définir au préalable pour bien se faire comprendre de son lectorat ${ }^{38}$. Le parti pris interprétatif des producteurs français s'aligne parfaitement sur l'objectif stratégique d'Arte, qui était de diversifier son

\footnotetext{
33 Nous utiliserons les deux coffrets DVD des premières saisons québécoise (Alliance Atlantis Viva film, 2005) et française (Arte, Making Prod, 2009). La deuxième saison de la série française a été diffusée entre le $1^{\text {er }}$ et le 22 février 2011 sur Arte et vient d'être commercialisée au format DVD.

34 Danielle Stanton, «François Létourneau, la revanche d'un nerd», Elle Québec, http://www.ellequebec.com/celebrites/franois-letourneau-la-revanche-d-un-nerd/a/25718, consulté le 30 juin 2011.

35 Dans le sillage des travaux de Véronique Nguyên-Duy sur le rôle de la presse dans les conditions de réception des séries télévisées, on peut se demander dans quelle mesure cette interprétation, que se sont efforcés de rejeter les scénaristes, n’a pas été véhiculée par l'institution médiatique.

${ }^{36}$ Making of de la série française (DVD, « bonus », 1'54”).

37 Pierre Sérisier, «Les Invincibles, quatre garçons dans la tourmente », blog Le Monde, 15 mars 2010, http://seriestv.blog.lemonde.fr/2010/03/15/les-invincibles-quatre-garcons-dans-latourmente/, consulté le 30 juin 2011 ; Isabelle Hanne, "'Les Invincibles', quatre garçons dans l'énervant ", Libération, 9 mars 2010, http://www.ecrans.fr/Les-Invincibles-quatregarcons, 9382.html, consulté le 30 juin 2011.

$3^{8}$ Louis-Bernard Robitaille, "Les Invincibles en France : des critiques déjà favorables », $L a$ Presse, 3 février 2010, http://www.cyberpresse.ca/arts/television/201002/03/01-945737-lesinvincibles-en-france-des-critiques-deja-favorables.php, consulté le 30 juin 2011.
} 
public en touchant spécifiquement le segment des trentenaires ${ }^{39}$. Cet objectif se reflète au niveau des choix de programmation des télédiffuseurs : tandis que la version québécoise est présentée de $21 \mathrm{~h}$ à $22 \mathrm{~h}$ à la suite de l'émission de divertissement grand public Le Match des étoiles (et juste avant le Téléjournal du soir), l'adaptation française est, elle, diffusée entre $22 \mathrm{~h} 20$ et $23 \mathrm{~h}$. On peut supposer que la deuxième partie de soirée constitue un créneau horaire particulièrement adapté au public des trentenaires, ne serait-ce que parce qu'il autorise une certaine liberté de ton ${ }^{\circ}$. En outre, il est intéressant de noter que la version française des Invincibles se trouve diffusée en alternance avec une autre série, Vénus et Apollon (Arte, 2005-), qui met en scène quatre femmes âgées entre 20 et 45 ans. Cette série apparaît en quelque sorte comme le contrepoint féminin des Invincibles dont la perspective est plutôt masculine, pour ne pas dire masculiniste. Le but visé par la chaîne semble atteint puisque, selon Emmanuel Suard, directeur des programmes, la part des téléspectateurs âgés entre 25 et 34 ans regardant Arte est passée de $0,5 \%$ à 2,5\% ${ }^{41}$. La chaîne a également largement tiré parti d'Internet et des médias sociaux pour se rapprocher de son public. En effet, au site Internet consacré à la série et proposant une variété de ressources s'ajoutent un site Facebook et de nombreux produits dérivés, comme les CD de la bande originale ainsi que du générique, chanté par le groupe La Chanson du Dimanche, dont fait partie Alexandre Castagnetti, coréalisateur de la version française. La série québécoise, pour sa part, avait bénéficié d'une campagne médiatique dont le point culminant fut le partenariat noué avec la compagnie de téléphonie Rogers. Celui-ci permettait aux abonnés de télécharger en exclusivité de courts extraits d'épisodes inédits. Ce partenariat a donné lieu à la diffusion de clips publicitaires mettant en scène de jeunes acteurs dont la tenue et l'aspect physique rappelaient étrangement ceux des protagonistes de la série ${ }^{42}$. L’intention commerciale avouée, en décalage avec le souhait initial des auteurs, était de cibler spécifiquement les 25-34 ans.

39 Contrairement à Radio-Canada, chaîne publique généraliste, Arte a l'image d'une chaîne « intellectuelle » et haut de gamme surtout reconnue pour la qualité de ses documentaires.

$4^{\circ 0}$ Néanmoins, la version française s'est débarrassée d'un certain nombre de scènes susceptibles de choquer, comme celle suggérant que Steve (Vince dans la version française) aurait eu une relation pédophile avec un jeune garçon.

${ }^{41}$ Emmanuel Suard, cité dans : Nathaëlle Morissette, « Les Invincibles français : Arte se satisfait des résultats ", La Presse, 8 avril 2010, lien consulté le 30 juin 2011, http://www.cyberpresse.ca/arts/television/201004/08/01-4268400-les-invincibles-francaisarte-se-satisfait-des-resultats.php.

${ }^{42}$ Ces clips publicitaires étaient présentés sans transition juste avant la coupure publicitaire à proprement parler, de sorte qu'ils semblaient faire partie intégrante de la série. 
En privilégiant le point de vue des «trentenaires », la version française a été conduite à opérer plusieurs changements par rapport à la série originale. En premier lieu, la volonté des producteurs de privilégier une distribution d'acteurs méconnus du grand public contribue, à notre avis, à renforcer l'exemplarité sociologique des personnages au détriment de leur individualité. Ainsi, François-Xavier (ou F-X) incarne le type même de l'intellectuel précaire préparant une thèse et n'ayant pas quitté le domicile familial. Vince, quant à lui, possède toutes les caractéristiques du nouveau riche, comme le reconnaissent les scénaristes Brigitte Bémol, Alexandre Castagnetti, Bertrand Marzec et Julien Simonet 43 . À l'inverse, les acteurs de la série québécoise bénéficiaient déjà d’une certaine notoriété que les scénaristes n'ont pas hésité à exploiter en jouant la carte de l'autoréférentialité. Le rôle de Rémi semble avoir été créé sur mesure pour Rémi-Pierre Paquin, guitariste et chanteur comme le personnage qu'il incarne, dont il partage même le prénom ${ }^{4}$. Par ailleurs, Pierre-François Legendre, Patrice Robitaille et Francois Létourneau avaient déjà eu l'occasion de jouer ensemble dans le long-métrage Québec-Montréal (réal. Ricardo Trogi, 2002), auquel le voyage en voiture au début de l'épisode 4 de la première saison fait explicitement allusion 45. Ce n'est pas le cas des acteurs français qui, outre leur relatif anonymat, n'avaient jamais encore tourné ensemble. À la différence donc de l'adaptation française, la série originale non seulement renforce l'identification entre les acteurs et leurs personnages, mais rappelle (pour ne pas dire commémore) intertextuellement leurs collaborations passées, inscrivant par là-même la série dans une histoire commune que l'on peut supposer connue des téléspectateurs québécois. Du point de vue de la réception, ce décalage se traduit par deux modes de relation différents aux héros de la série. Tandis que les spectateurs québécois auraient davantage tendance à se faire les commentateurs voire les juges des agissements des protagonistes (on pourrait parler d'un effet de distanciation, que contribue à accentuer l'usage de plans larges), les spectateurs français, quant à eux, tendraient plutôt à se retrouver dans les personnages, ce que renforce le choix de la

43 Voir Lucas Armati, « Vive le Québec annexé ! », Télérama, 10 mars 2010, p. 86.

44 On pourrait en dire de même du rôle de P-A, campé par François Létourneau, qui est lui-même titulaire d'une maîtrise en philosophie et passe pour être un intellectuel dans le paysage artistique québécois, un « nerd » pour reprendre le titre de l'article de Danielle Stanton.

45 On notera également la présence de Stéphane Breton (dans le rôle de Martin), qui incarnait Rivard dans Québec-Montréal. 
stratégie de programmation (on pourrait, par opposition, parler d'un effet d'identification, favorisé par l'utilisation de plans rapprochés) ${ }^{46}$.

On peut aussi souligner que plusieurs personnages secondaires de la version française jouissent d'une grande notoriété, qui contraste avec celle des acteurs principaux ${ }^{47}$. C'est le cas de Clémentine Célarié (absente dans la deuxième saison), de François Rollin, de Marie-Christine Adam et de Jackie Berroyer, qui tiennent respectivement les rôles de Gisèle, du père Poitras, de Thérèse et de Paul Boisvert. Représentants de la génération des parents baby-boomers, ces acteurs établis confèrent davantage de crédit et de «traçabilité » à la série. On notera que le couple composé par MarieChristine Adam/Thérèse Boisvert et Jackie Berroyer/Paul Boisvert est beaucoup plus polarisé et dysfonctionnel que dans la version originale, ce qui permet d'exploiter davantage le talent comique des comédiens, parfois sur le mode de la caricature.

En prenant le parti de cibler la génération des trentenaires, la version française fait planer un parfum de nostalgie qui se manifeste par un certain nombre de choix stylistiques touchant en particulier le générique. Il existe deux versions du générique français: une version courte, en ouverture de chaque épisode, dont la forme rappelle beaucoup l'original, et une version longue, qui est en fait le clip accompagnant le single chanté par La Chanson du Dimanche. Les paroles relativement triviales de l'une et de l'autre version se limitent à une juxtaposition de phrases où la rime l'emporte à l'évidence sur la teneur du message (c'est en particulier le cas du refrain : "Quatre héros, des temps nouveaux. Les Invincibles. Quatre copains, doigts de la main. »). Elles rappellent, de même que la musique qui les accompagne, les génériques des dessins animés japonais qui ont fait le bonheur des trentenaires dans leur jeunesse ${ }^{48}$. Fortement influencée par l'imaginaire décalé de $\mathrm{La}$ Chanson du Dimanche, la version longue se caractérise par son second degré. Le retour régressif à l'enfance s'opère sur un fond d'autodérision 49 dont rendent compte à la fois les paroles de la

${ }^{46}$ En toute rigueur, il faudrait procéder à une comparaison systématique des réactions des spectateurs québécois et français sur les forums consacrés à la série.

$47 \mathrm{La}$ version québécoise bénéficie également d'un casting haut de gamme avec la présence de Louise Bombardier (Gisèle), Donald Pilon (M. Boivert) et même Robert Lepage, dans la troisième saison.

${ }^{48}$ Les publicitaires ont su exploiter cette tendance, comme en témoigne, par exemple, la sortie récente du DVD «Mes années 1980 », consacré à Albator, Capitaine Flam et Les Mystérieuses Cités d'or.

${ }^{49}$ Le second degré est l'une des marques distinctives de Making Prod, qui a coproduit la version française des Invincibles. On doit à Making Prod Empreintes criminelles, parodie de CSI diffusée sur France 2 entre le 25 mars 2011 et $1^{\mathrm{er}}$ avril 2011 et Putain de série !, diffusée sur Dailymotion entre 2008 et 2009, qui parodie cette fois le travail de scénaristes. 
chanson, les costumes et la chorégraphie des chanteurs, ce qui le rend sans doute plus acceptable. Deux strophes de la chanson communiquent sans ambiguïté cette nostalgie exacerbée : "Ils rêvent d'avoir tous les jours vingt ans,/ d'avoir le pouvoir de vaincre le temps $5^{\circ}$ ». La version courte est plus proche de l'esprit du générique de la série originale, mais contient néanmoins un certain nombre d'ajouts. En premier lieu, elle associe des couleurs spécifiques aux personnages, à la manière des Power Rangers. À ce propos, on retrouvera tout le long de la version française des références à cet univers graphique japonais, que ce soit à travers les figurines de Dragon Ball $Z$ dans la chambre d'Hassan ou bien par certains traits narratifs et graphiques de la bande dessinée animée dont ce dernier est l'auteur. En second lieu, le générique français (voir figure 1) précise davantage le profil psychologique des protagonistes, conformément au souhait des scénaristes français ${ }^{5}$. Le premier personnage est Hassan, force bleue, qui dessine son propre personnage, "Alias », ce qui renseigne sur sa passion pour la bande dessinée et introduit le principe de duplicité qui le caractérise. Suit F-X, force verte, marchant seul dans le désert, un petit sourire crispé sur le visage. Vient ensuite Vince, force rouge, l'air sérieux et placé à gauche de la silhouette d'une femme nue, ce qui met immédiatement l'accent sur sa libido. Finalement, Mano, force jaune, fait un doigt d'honneur qui témoigne de son état de perpétuelle rébellion. On note également la présence d'un dessin de cour entouré de notes de musique, référence au rêve de Mano d'être une star de rock. Dans la version québécoise (voir figure 2), la référence aux comics est pleinement assumée et se suffit à elle-même; elle ne nécessite ni l'intervention d'un (méta)récit chanté, ni l'ajout d'attributs ou de symboles particuliers qui renseigneraient sur le profil des personnages. L'unité du médium graphique crée, de façon plus économique, un lien entre le portrait monochrome des personnages, leurs avatars et leur double identité.

$5^{\circ}$ La première de ces strophes fait étrangement écho par son contenu au titre du standard de Berthe Sylva « On n’a pas tous les jours vingt ans ».

${ }^{51}$ Armati, p. 86. 


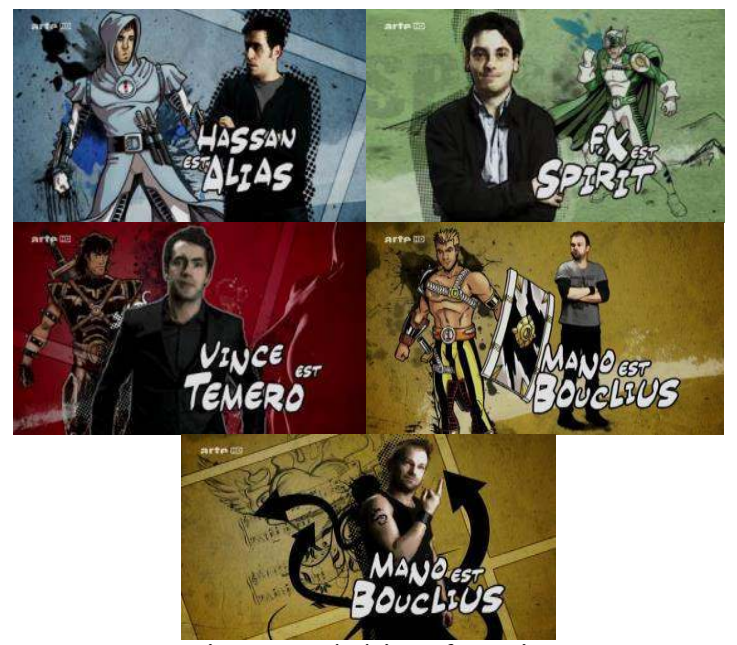

Fig. 1 : Le générique français.

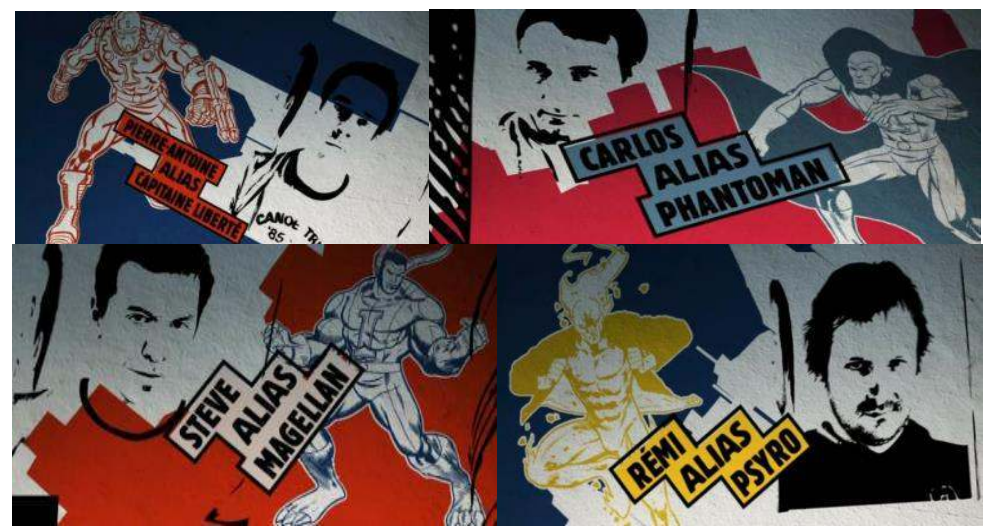

Fig. 2 : Le générique québécois.

b) Opacité historique vs transparence contemporaine

L'une des particularités de la série est de faire appel à des effets spéciaux dans des situations souvent très ordinaires. C'est le personnage de Carlos/Hassan qui concentre l'essentiel de ces effets spéciaux - souvent 
filmés en ocularisation interne. Plus précisément, la paranoïa de Carlos/Hassan à l'endroit de sa compagne alimente un fantasme de persécution dans lequel le père de cette dernière semble vouloir avertir son futur gendre d'un danger imminent que l'on devine être en lien avec le mariage à venir ${ }^{52}$. Bien plus que leur efficacité, ce qui caractérise les effets spéciaux de la version québécoise est leur « effet de matérialité passé53 ». Ceux-ci se manifestent comme autant de citations intertextuelles tirées de séries (Les Envahisseurs, V, Star Trek) et de films (Terminator, Scream) des années 1980-90, dont ils conservent la facture visuelle (voir figure 3). En d'autres termes, les effets spéciaux de la version québécoise portent la mémoire matérielle d'une certaine tradition filmique (science-fiction, slasher movies) qui alimente de multiples renvois intertextuels.

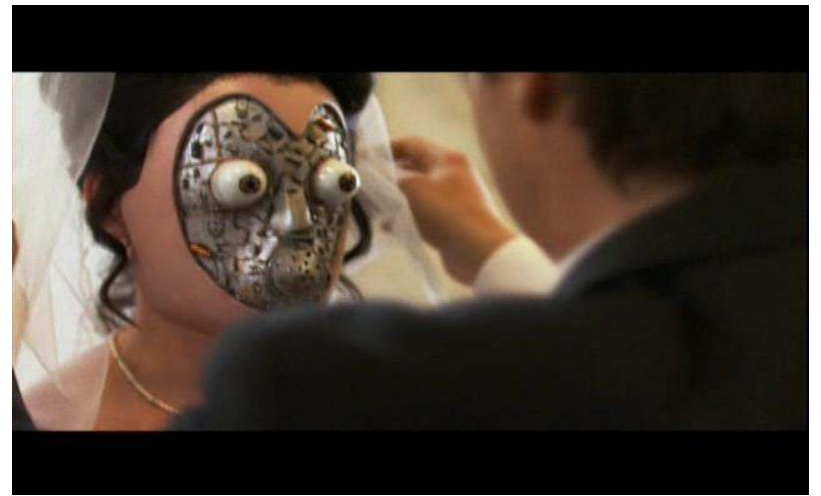

Fig. 3 : Lyne le robot, référence à Terminator dans la version québécoise.

Ce n'est pas le cas des effets spéciaux de la version française, qui utilisent des procédés techniques plus contemporains, et donc moins visibles, comme les séquences (auto)animées qui remplacent les planches de comics de la série originale, animées quant à elles à distance, par des mouvements

$5^{2}$ On peut voir dans ce leitmotiv une référence à la théorie du complot à l'œuvre dans de multiples séries étasuniennes comme The X-Files (Fox, 1993-2002).

53 Dans son ouvrage intitulé Télévision. Réalité ou réalisme (Bruxelles, De Boeck \& Larcier, INA, 2002), Muriel Hanot définit l'effet de matérialité passé comme la « résultante du jugement de matérialité exercé au niveau plastique de la matière télévisuelle, qui inscrit le message télévisuel en porte à faux par rapport aux normes habituelles de la réception, grâce à des indices tels le noir et blanc, la rupture du suivi de photogrammes, des parasites..., induisant ainsi une impression de passé. » (p. 54) 
de caméra. S'ils assurent incontestablement une meilleure transition visuelle entre la réalité de la diégèse et la fiction du dessin, les effets spéciaux de la version française contribuent cependant à donner une plus grande autonomie à la séquence animée, ce qu'ont également noté Pierre Sérisier et Isabelle Hanne ${ }^{54}$. Trois éléments en particulier renforcent cette autonomie par rapport à la diégèse : contrairement à la planche de comics, la séquence animée ne porte pas les traces matérielles (coups de crayons, ratures) de l'intervention d'Hassan ; les voix des alter ego graphiques des personnages sont indépendantes de la diégèse, alors qu'elles sont toutes jouées par Carlos dans la série québécoise; le scénario de la séquence animée est d'une plus grande complexité (terminologique, narrative) que celui du comic book de la version originale. La séquence graphique de la version française semble ainsi coupée matériellement de son créateur et prendre vie par elle-même.

En outre, la série française multiplie les références à l'esthétique transparente de l'horreur (voir figure 4). Si l'empreinte du genre horrifique est omniprésente dans la version française - ce qui lui assure une certaine cohérence visuelle, intertextuelle et même thématique55 -, elle cohabite avec d'autres genres dans la série québécoise, tels le fantastique et même le surréalisme, comme l'illustre la scène où Carlos imagine une conversation entre deux poulets ( $\left.\mathrm{VQ}^{56}: 1.3 / 25{ }^{\prime}{ }^{\prime} 00^{\prime}\right)$.

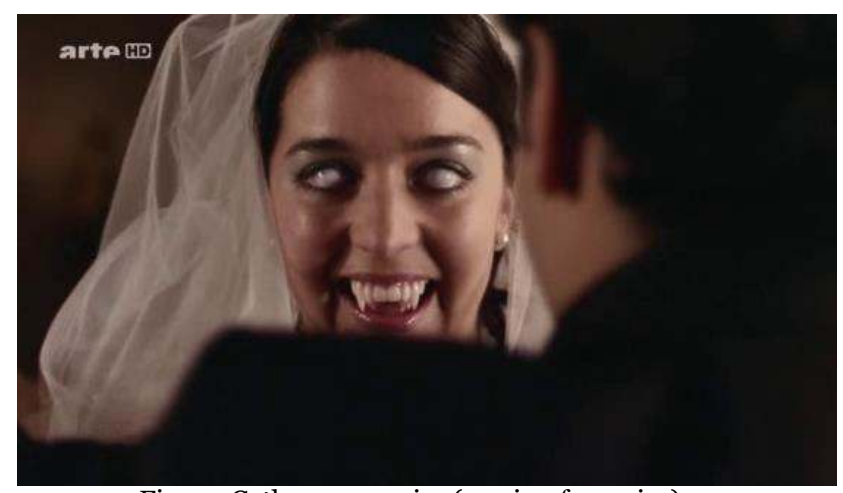

Fig. 4 : Cathy en vampire (version française).

54 Dans les articles du Monde et de Libération cités précédemment.

55 La figure du vampire suceur de sang rend particulièrement bien compte du comportement possessif et castrateur de Cathy, qui est montrée à plusieurs reprises en possession d'un couteau.

${ }^{56}$ Nous utiliserons les abréviations VQ et VF pour désigner respectivement la version québécoise et la version française. 


\section{c) Retrouvailles vs fiction des origines}

Le plan d'ouverture du premier épisode de la version française est une photo de jeunesse des quatre héros, ce qui contribue à ancrer dès le début les personnages dans une histoire longue antérieure au début du récit. En revanche, la série originale s'ouvre sur un flash-back d'une portée beaucoup plus courte (pas plus de quelques semaines avant la scène du pacte) mais d'une amplitude beaucoup plus grande (plusieurs minutes). Le contenu de cette analepse a la particularité de mêler réalité et fiction 57 , mais aussi de fonctionner comme bande-annonce de la série. Utilisant un montage en accéléré accompagné d'une musique dramatique, elle suscite un effet d'attente non pas tant sur la nature du lien qui unit les personnages que sur le motif de leurs retrouvailles imminentes, motif sur lequel on maintient volontairement le doute $5^{8}$. Partant, l'histoire d'amitié entre les personnages se construit au fur et à mesure de la diégèse par le biais de flash-back, de renvois intertextuels à leurs collaborations passées ou tout simplement au gré de témoignages, lors des séquences en "caméra vérité ». Les réalisateurs français, pour leur part, ont eu la volonté d'authentifier le lien unissant les protagonistes par la fiction d'une enfance commune, à laquelle le support photographique (un montage, bien sûr) confère un semblant de réalité. Peut-être faut-il voir là une tentative de compenser l'absence de relations antérieures connues des téléspectateurs français entre les quatre acteurs principaux. C'est donc la fiction, posée comme authentification d'une histoire commune, qui prend entièrement en charge la mise en scène de ce lien d'amitié entre les acteurs-personnages français, lequel se consolidera dans l'expérience vécue du tournage ${ }^{59}$. Du point de vue iconique, ce lien s'établit par le parallélisme visuel entre la photo représentant les quatre protagonistes enfants et le plan qui suit où ils sont tous assis, adultes, autour d'une table, le mouvement de la caméra figurant le temps qui s'est écoulé. En somme, au suspense entourant le motif des retrouvailles dans la version québécoise se trouve substituée, dans la version française, la fiction d'une enfance commune. D’un point de

57 La séquence comprend une image à valeur métadiégétique faisant référence au tournage même, de sorte que s'opère une confusion sciemment entretenue entre la participation des acteurs au tournage de la série et celle des personnages au pacte. Cet effet de métalepse renforce encore davantage l'identification entre acteurs réels et personnages de fiction.

$5^{8}$ À ce propos, il convient de remarquer que, dans l'analepse, les quatre acteurs ne sont pas présents simultanément à l'écran. C'est le montage sur la base d'un raccord-objet, en l'occurrence un téléphone portable, qui établira un lien entre les personnages.

59 Le making of de la version française fait largement état de la façon dont les acteurs ont développé un lien d'amitié au fil du tournage, lien qui s'est trouvé réinvesti dans la fiction. 
vue narratif, cette fiction permet de régler a priori la question du lien unissant les acteurs-personnages.

\section{d) Prendre son temps ou bien passer à l'action}

Sur le plan de la narration, les adaptateurs français ont dû négocier avec la différence de format entre les séries française et québécoise. Tandis que la première saison de la version originale comporte 12 épisodes de 44 minutes (soit 528 minutes au total), la version française est construite en 8 épisodes de 52 minutes (soit 416 minutes au total). Cette contrainte a donné lieu à des réaménagements considérables dans la diégèse. Les épisodes de la version française sont ainsi beaucoup plus condensés que ceux de la version originale. À titre d'exemple, le périple des quatre héros à la campagne, qui durait un épisode entier dans la version originale, se trouve réduit à une vingtaine de minutes dans l'adaptation. Le choix des adaptateurs français semble avoir été de privilégier l'action au détriment de l'ambiance (qui, de ce point de vue, s'apparente à des longueurs), tout en minimisant autant que possible les intrigues secondaires.

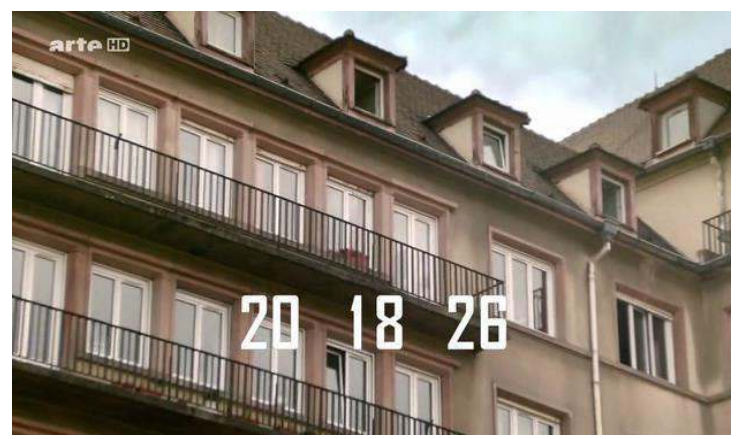

Fig. 5 : Les compteurs dans la version française.

L'utilisation des compteurs incrustés à la manière de 24 Heures chrono (voir figure 5) a pour effet d'orienter quasi-exclusivement le récit vers la réalisation des engagements pris lors des pactes. Nous verrons que ce choix entraîne une certaine perte de qualité esthétique dans la version française. 


\section{La question de l'espace}

Les scénaristes français disent avoir cherché à «verrouiller60 » la psychologie des personnages. Pour ce faire, ils ont limité le recours au comique de situation, très présent, selon eux, dans la version québécoise, tout en conférant aux personnages une plus grande épaisseur psychologique. Prenant ce constat à rebours, nous montrerons que la dimension psychologique, si elle est certes peu visible au niveau de la diégèse et de la performance d'acteur dans la série québécoise, est intimement liée à la figuration de l'espace.

\section{a) La psychologie par l'image}

Dans la version québécoise, l'exploitation de la profondeur de champ combinée à celle de la distance focale permet une grande variation au niveau des échelles de plan, du gros plan au plan d'ensemble. Ce n'est pas le cas de la version française, qui fonctionne avec une variété de plans relativement limitée, allant du plan moyen au plan rapproché. L'usage plus fréquent dans la série originale de plans moyens (plans narratifs, plans américains) et de plans d'ensemble permet de mieux marquer le lien entre les personnages et leur environnement, lequel est rendu encore plus visible du fait de ses couleurs vives (qui rappellent le chromatisme primaire des comics). Les personnages sont non seulement chromatiquement assortis à leur espace (lieu de résidence, lieu de travail), mais font littéralement corps avec celui-ci (voir figures 6 et 7).

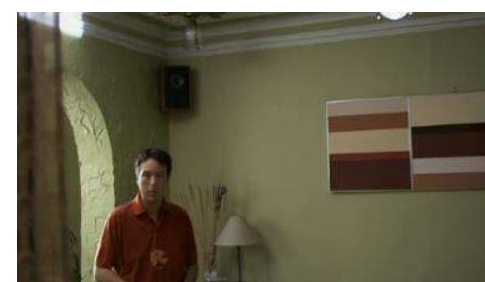

Fig. 6 : Carlos (version québécoise).

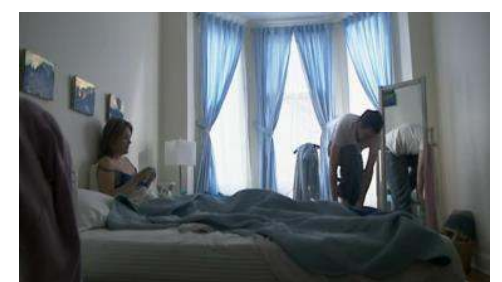

Fig. 7 : P-A et Vicky (version québécoise).

De nombreux indices profilmiques, comme les posters, les tableaux (faisant parfois l'objet d'inserts) ou la décoration d'intérieur donnent autant d'indications sur l'identité sociale et culturelle des personnages. Dans la version française, ce lien est particulièrement manifeste dans le cas

${ }^{60}$ Armati, p. 86. 
du personnage de Cathy, souvent filmée dans l'intérieur rose bonbon de la chambre du couple, où elle complote pour assouvir ses rêves de mariage (voir figure 8).

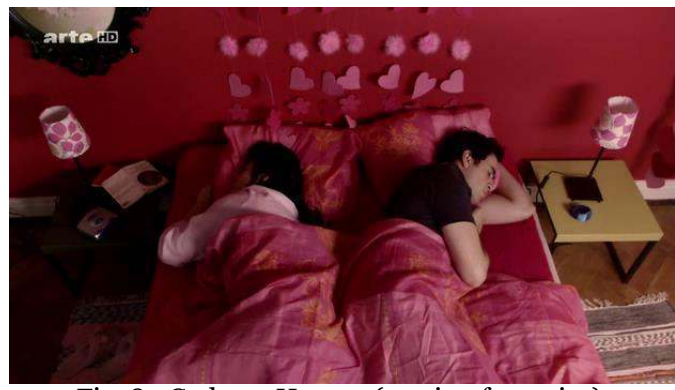

Fig. 8 : Cathy et Hassan (version française).

Sur le plan narratif, l'attachement des personnages à leur lieu de vie est particulièrement marqué. Le cas le plus symptomatique (pour ne pas dire pathologique) est celui de $\mathrm{P}-\mathrm{A}$, qui est incapable d'aller à la selle en dehors de chez lui. Quant à l'atelier de Carlos, il constitue le seul espace de liberté où il peut échapper à la surveillance de Lyne. L'intérieur bourgeois de Steve renseigne à la fois sur son statut social, mais aussi sur ses névroses, en particulier sa propreté maladive (dont on peut penser qu'elle sert d'écran à ses travers sexuels). En outre, les séquences de témoignage inspirées par l'esthétique du mockumentaire contribuent encore davantage à associer les personnages à l'intimité de leur lieu de vie, où ces entretiens se déroulent. Par contraste, le chromatisme souvent plus terne des décors et l'utilisation fréquente de plans rapprochés dans la version française restreignent l'identification entre le personnage et son espace, bien que la cartographie générale des lieux soit par ailleurs maintenue. Il en est de même de la suppression, probablement due à l'impératif de condensation, de plusieurs scènes domestiques (petit déjeuner, brossage de dents) qui ancraient davantage les personnages dans leur environnement de tous les jours et rappelaient ainsi la scénographie du quotidien des téléromans. D’une manière générale, l'impression d'espace varie énormément entre la série originale et son adaptation. Comprimé dans cette dernière au point où les acteurs sont parfois pratiquement l'un derrière l'autre (VF : 1.1/17'34"), l'espace paraît plus ouvert dans la version québécoise pour les raisons évoquées précédemment, ce qui offre l'avantage de multiplier les possibilités de déplacements, tant visuels que physiques. 
Nous soutiendrons que, loin d'être absente ou défaillante dans la série québécoise, la dimension psychologique se met en scène au niveau stylistique et, plus spécifiquement, au niveau iconique plutôt qu'au niveau du jeu d'acteur ou de la trame narrative. À cet égard, plusieurs plans sont particulièrement remarquables. C'est le cas d'une série de plans décentrés qui sont consécutifs aux ruptures des héros, plans qui se trouvent liés par le montage. Reléguer l'acteur aux marges du plan crée un vide que l'on peut interpréter tantôt comme le signe d'une absence, tantôt comme l'empreinte vivace laissée par une présence forte (voir figures 9 et 10).

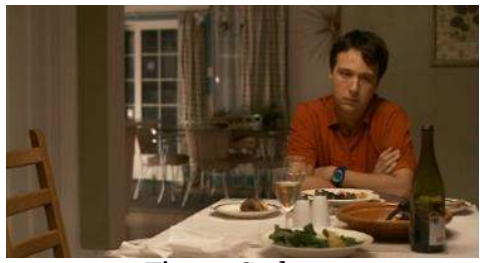

Fig. 9 : Carlos.

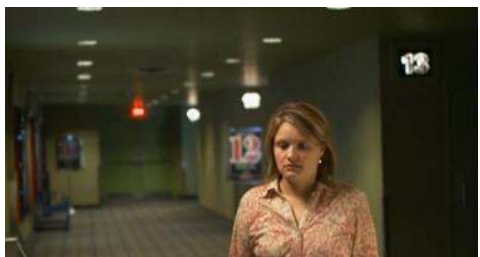

Fig. 10 : Vicky.

Ce langage visuel a disparu de la version française, qui fait le choix de restituer l'action au détriment de l'ambiance, comme nous l'avons souligné précédemment. C'est davantage sur le visage des acteurs filmés en plans serrés que dans leur positionnement dans le champ de la caméra que se lisent les émotions. Un autre exemple intéressant est le plan de demiensemble sur Kathleen (VQ : 1.1/38'42") figurant tout son accablement tandis que sa grand-mère agonise et que Steve vient de rompre. L'amplitude du plan couplée à la profondeur de champ perd littéralement Kathleen qui, debout au second plan, finit par s'effondrer dans son fauteuil pour être avalée dans l'arrière-plan. Cette disparition se trouve renforcée visuellement par l'éclairage sombre de la chambre d'hôpital. Dans la version française, la scène ne fait l'objet d'aucune stylisation particulière. D'une manière générale, dans la série originale, l'utilisation de la profondeur combinée au changement d'échelle de plan et à l'exploitation du chromatisme produit une idiosyncrasie stylistique très riche permettant de véhiculer une variété d'émotions. Mise à profit dans des espaces intérieurs, la profondeur de champ permet à la fois d'utiliser les cadres naturels que sont les seuils de porte et les fenêtres pour décloisonner l'espace, mais aussi de tirer parti des couloirs, qui deviennent alors autant d'axes privilégiés de déplacements. 


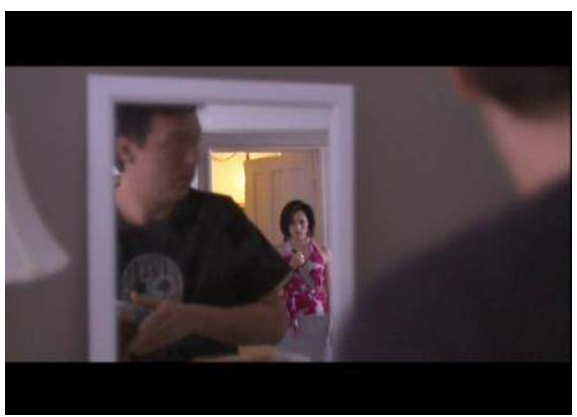

Fig. 11 : Carlos et Lyne (version québécoise).

La scène de l'épisode 4 où Carlos se cache de Lyne est particulièrement emblématique. À la façon des slasher movies les plus classiques du genre (Psychose, Scream), la série utilise les cadres naturels (seuil de la porte et bord du miroir) combinés à la profondeur de champ pour créer un effet de mise en abyme. L'utilisation du miroir comme dispositif iconique de réflexion renforce encore davantage l'aspect irréel de l'apparition de Lyne (voir figure 11). Par comparaison, la version française recourt à une mise en scène moins sophistiquée misant sur le champ/horschamp et l'emploi d'une musique dissonante (voir figure 12).

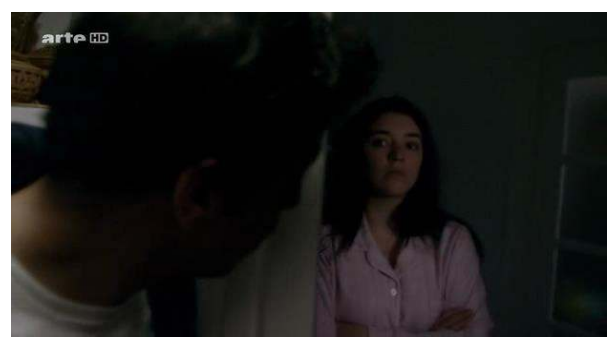

Fig. 12 : Hassan et Cathy (version française).

Dans un registre similaire, l'emploi de la profondeur de champ permet de faire entrer l'amant de Jolène dans le cadre en arrière-plan (VQ : $\left.1.1 / 27^{\prime} 44^{\prime \prime}\right)$, ce qui constitue un indice supplémentaire préfigurant la rupture et confirmant les doutes des téléspectateurs sur l'identité de l'amant en question. Dans la version française, cette rupture s'opère de façon plus brutale sans faire l'objet d'un développement narratif particulier, là encore peut-être en raison des contraintes de temps. 
b) De Montréal à Strasbourg

Plutôt que de se dérouler à Paris comme on aurait pu s'y attendre, la version française se passe à Strasbourg. Les réalisateurs justifient ce choix par leur volonté de faire référence à l'identité européenne d'Arte et au fait qu'Arte diffuse également en Allemagne ${ }^{61}$. À l'image de Montréal, Strasbourg est une ville à la fois bilingue (on y parle français et allemand) et cosmopolite, ce dont rendent compte plusieurs éléments référentiels : dans le premier épisode, F-X et Meike vont au cinéma voir un film étranger en langue allemande; dans le deuxième épisode, on apprend que Vince travaille comme responsable de communication au Parlement européen. Quant à Hassan, il a la particularité d'être à moitié turc (et non plus à moitié espagnol comme dans la série originale), ce qui est conforme à la réalité sociologique de l'immigration locale. Cela dit, dans l'ensemble, la série reste beaucoup plus française qu'européenne. Au skyline de Montréal qui ouvre le générique de la version québécoise renvoie le zoom sur les quatre protagonistes aux abords de la place de l'Homme de Fer à Strasbourg. Pour les spectateurs français qui connaissent mal la métropole strasbourgeoise, il est certain que les multiples plans fixes de la ville jalonnant les épisodes peuvent donner une impression d'étrangeté. Néanmoins, on peut penser que cette impression se dissipe en partie du fait de la présence, dans ces plans fixes, de signifiants (immeubles, transports, rues bondées) qui renvoient à l'idée générique d'urbanité. Pour ce qui est de la série québécoise, elle se déroule dans un espace bien connu des spectateurs québécois, à savoir le Montréal francophone.

\section{Conclusion}

Sans conteste, les scénaristes ont réussi leur pari de transposer Les Invincibles dans le contexte français. Nécessaire compte tenu des écarts culturels et linguistiques avec le Québec, cette transposition s'est parfois accompagnée d'une bonne dose de créativité. Par exemple, alors que P-A compare le pacte entre les quatre amis à la Déclaration d'indépendance des États-Unis au début du premier épisode de la série originale, c'est la Déclaration des droits de l'homme et du citoyen qui est mentionnée dans l'adaptation française. Cette adaptation culturelle autorise une allusion fort bienvenue à l'échec du projet révolutionnaire de Marat et de Danton. Celleci assoit alors - de tout le poids de l'histoire de France - la mise en garde formulée par Hassan au moment de l'adoption du pacte. Outre la différence

${ }^{61}$ Armati, p. 86. 
de format, qui a conduit à des aménagements majeurs au niveau de la diégèse, l'une des contraintes significatives de l'adaptation aura été de négocier avec la part d'américanité de l'identité culturelle québécoise. Polymorphe, cette américanité se manifeste au niveau de la langue, des morceaux choisis pour la bande-son, de la référence aux comics Marvel ou encore de l'intertexte cinématographique et télévisuel. Les adaptateurs ont souvent trouvé des solutions originales et efficaces pour transposer cette américanité : la bande-son fait davantage de place à des standards du rock britannique, la culture manga bien connue des trentenaires se trouve substituée à celle des comics et l'intertextualité se renouvelle sous la forme d'emprunts au genre de l'horreur et à d'autres séries télévisées comme 24 Heures chrono. L'ensemble de ces éléments contribue à faire des Invincibles une série singulière dans le paysage télévisuel français. De notre point de vue, c'est essentiellement au niveau de la dimension iconique que l'original se distingue de l'adaptation. Cette distinction relève moins d'un jugement de valeur que de la prise en compte de la singularité du langage cinématographique mis en œuvre dans la série québécoise, ainsi que nous l'avons montré. Compte tenu de son originalité, un tel langage se prête moins à une adaptation qu'à un véritable travail de recréation. Il n'en reste pas moins que la version française reprend à son compte un certain nombre d'éléments de ce langage (écrans divisés, soin porté aux raccords, utilisation de plans-séquences, jeu de plongée/contreplongée marquant le rapport de force entre Cathy et Hassan). Enfin, nous ne pourrions pas conclure sans évoquer les multiples clins d'œil intertextuels que fait la version française à l'original. Ainsi, dans le premier épisode, Vince mentionne le nom de Sandra, qui se trouve être celui de l'une des protagonistes de la série originale. Pour sa part, Cathy parle d'une de ses connaissances d'origine espagnole ne sachant pas faire la paella. Il s'agit sans doute d'une allusion au personnage de Carlos, qui, dans la série québécoise, est lui-même à moitié espagnol et se targue d'être un spécialiste de la paella. Dans le sixième épisode, F-X, parlant de son excompagne, s'adresse à Mano en français québécois : "J'aurais préféré qu'elle parte au Québec, calice ce s'rait l'fun. » Enfin, dans le dernier épisode de la saison, Pierre-François Legendre (Pierre-Alexandre dans la version québécoise) fait une brève apparition sous les traits du docteur qui fait accoucher Jeanne.

En définitive, l'adaptation française des Invincibles s'avère être un pari réussi en ce sens qu'elle a sans aucun doute renouvelé l'imaginaire télévisuel hexagonal en conservant une part de l'étrangeté originelle de la série québécoise (construction en trois niveaux diégétiques, langage cinématographique, cohabitation de plusieurs supports médiatiques). Dans 
une optique comparative, il serait intéressant de voir si les autres adaptations françaises de séries québécoises, à l'instar d'Un gars, une fille et Des Bougon, connaissent la même réussite.

\section{Bibliographie}

AUBRY Danielle, "Réflexion sur les genres télévisuels », Fréquence/Frequency, ${ }^{\circ} 11-12,2004$, p. 92-112.

ARMATI Lucas, «Vive le Québec annexé! », Télérama, 10 mars 2010, p. 86.

BARRETTE Pierre, "L'état de la série télé au Québec : petite enquête en forme de dialogues », 24 images, $\mathrm{n}^{\circ} 138$, 2008, p. 18-20.

BEEDEN Alexandra et Joost de BRUIN, "The Office: Articulations of National Identity in Television Format Adaptation ", Television \& New Media, vol. 11, $\mathrm{n}^{\mathrm{o}} 1$, juin 2010, p. 3-19.

BERDOT Françoise, La télévision d'auteur(s). Aléas éditeur, Lyon, 20032004.

BOUCHARD Nathalie Nicole, «À la recherche des téléromans : revue de la littérature consacrée aux radioromans et aux téléromans québécois ", Communication, vol. 20, $\mathrm{n}^{\circ} 1$, 2000, p. 217-248.

BOUCHARD Nathalie Nicole, «Scoop et les communautés interprétatives : Sémiotique de la réception du téléroman québécois ", Thèse de doctorat en communication, Montréal, UQAM, 1997.

Commissariat aux langues officielles, Ombres sur le paysage télévisuel canadien: place du français sur les ondes et production en contexte minoritaire, 2009,

http://www.ocol-clo.gc.ca/docs/f/televisionlandscape paysagetelevisuel f.pdf, consulté le 30 juin 2011

DE LA GARDE Roger, « Le téléroman québécois : une aventure américaine », Ciberlegenda, $\mathrm{n}^{\circ}$ 10, 2002, http://www.uff.br/mestcii/roger1.htm, consulté le 30 juin 2011. 
DEMERS Frédéric, «Téléroman, télésérie, feuilleton... : retour sur une source de confusion sémantique », Communication, vol. 25, $\mathrm{n}^{\circ}{ }^{1}, 2006$, http://communication.revues.org/index1480.html, consulté le 17 mai 2011.

GAUTHIER Philippe, «Les coproductions cinématographiques FranceQuébec : un espace culturel problématique. L'exemple de J'ai mon voyage ! (Denis Héroux, 1973). Nouvelles 'vues' sur le cinéma québécois ", Cinéma québécois, $\mathrm{n}^{\circ} 10$, 2008-2009, p. 1-16.

GOUDREAU Nathalie, «Les téléromans au Québec en 1993-1994 et 19941995. Contenu, conditions de production et auditoire », Communication, vol. $18, \mathrm{n}^{\circ} 1,1997$, p. 125-136.

HANNE Isabelle, “'Les Invincibles', quatre garçons dans l'énervant », Libération, 9 mars 2010, http://www.ecrans.fr/Les-Invincibles-quatregarcons,9382.html, consulté le 30 juin 2011.

HANOT Muriel, Télévision. Réalité ou réalisme, Bruxelles, De Boeck \& Larcier, INA, 2002.

HUTCHEON Linda, Theory of Adaptation, New York et Londres, Routledge, 2006.

LEROY Dominic, « Téléroman québécois et identité culturelle nationale », Études canadiennes, $\mathrm{n}^{\circ} 34,1993$, p. 7-27.

MORAN Albert, TV Formats Worldwide: Localizing Global Programs, Bristol et Wilmington, Intellect Books, 2009.

MORISSETTE Nathaëlle, «Les Invincibles français: Arte se satisfait des résultats ", La Presse, 8 avril 2010, http://www.cyberpresse.ca/arts/television/201004/08/01-4268400-lesinvincibles-francais-arte-se-satisfait-des-resultats.php, consulté le 30 juin 2011.

NGUYÊN-DUY Véronique, "Le téléroman et la volonté d'une télévision originale », in Variations sur l'influence culturelle américaine, éd. Florian Sauvageau, Sainte-Foy, Québec, Les Presses de l'Université Laval, 1999, p. 131-158. 
ROBITAILLE Louis-Bernard, «Les Invincibles en France : des critiques déjà favorables ", La Presse, 3 février 2010,

http://www.cyberpresse.ca/arts/television/201002/03/01-945737-lesinvincibles-en-france-des-critiques-deja-favorables.php, consulté le 30 juin 2011.

SAOUTER Catherine, «Le téléroman, art de nouveaux conteurs : formes et influences du récit téléromanesque », Recherches sociographiques, vol. 33, $\mathrm{n}^{\circ} 2,1992$, p. 259-276.

SÉRISIER Pierre, "Les Invincibles, quatre garçons dans la tourmente » blog Le Monde, 15 mars 2010,

http://seriestv.blog.lemonde.fr/2010/03/15/les-invincibles-quatregarcons-dans-la-tourmente/, consulté le 30juin 2011.

STANTON Danielle, "François Létourneau, la revanche d'un nerd », Elle Québec, http://www.ellequebec.com/celebrites/franois-letourneau-larevanche-d-un-nerd/a/25718, consulté le 30 juin 2011. 